\title{
Postoperative pancreatic fistula after pancreatic resection
}

\author{
Sabol M, Donat R, Dyttert D, Reken V, Sintal D, Palaj J, Durdik S \\ St. Elizabeth Institute of Oncology, Bratislava, Slovakia. martin.sabol@ousa.sk
}

\begin{abstract}
BACKGROUND: The failure of pancreatic anastomosis after the proximal pancreaticoduodenectomy (PD) and the failure of pancreatic stump after the distal pancreatectomy with a resulting postoperative pancreatic fistula remain the most feared complications after pancreatic resection. Surgeons have been trying to find a reliable reconstructive technique of pancreatic anastomosis for decades.

METHODS: A literature search was performed to January 2020. Studies giving a detailed description of the pancreatic anastomosis after open PD and pancreatic stump closure techniques after the distal pancreatectomy were included. The aim of this study was review reported data derived from meta-analyses concerning the incidence of POPF according to the International Study Group of Pancreatic Surgery. A comparison of various surgical techniques and their impact on POPF incidence was made.

RESULTS: In the group of clinically relevant POPF (CR- POPF), a well established difference between the patients undergoing POPF-associated interventional drainage or reoperation was observed. Meta-analyses showed that the patients with CR- POPF were statistically more likely to have a small duct size, soft gland texture, particular pancreatic neoplasms and an excessive intraoperative blood loss.

CONCLUSION: Grade C POPF following PD, although uncommon, occurs with a defined incidence and is associated with a substantial morbidity, prolonged hospitalization, delayed recovery and a significant mortality. According to the results of various meta-analyses, pancreatogastrostomy and pancreatojejunostomy seemed to be comparable anastomotic techniques following PD (Ref. 54). Text in PDF www.elis.sk KEY WORDS: pancreatic fistula, pancreaticoduodenectomy, distal pancreatectomy.
\end{abstract}

\section{Update of definition and grading of POPF}

The International Study Group of Pancreatic Fistula (ISGPF) developed in 2005 a definition of postoperative pancreatic fistula. Since its definition, it has reached a global acceptance. Numerous articles validated the ISGPF definition and grading of POPF. While the mortality rates following a pancreatic surgery have come down to less than $5 \%$, the morbidity still remains high, ranging between $30-50 \%$, even in the high volume centres (1). Pancreatic fistulas are among the most common complications after PD occurring in $18 \%$ of patients (2).

A decreased rate of complications can be attributable to concentration in high volume, specialized HPB centres, innovation of surgical techniques and perioperative management.

Requirements for an update of the definition and grading of POPF have become imperative, and as the result, the update of the International Study Group definition and grading of POPF was published in 2016, 11 years after an original definition 2016 (3) The consequences of POPF following a pancreatic resection belong to either "clinically relevant” fistulas ,grades B-C“ or ,harmless“ grade A fistula” or „no fistula“ at all. According to the updated

St. Elizabeth Institute of Oncology, Bratislava, Slovakia.

Address for correspondence: $\mathrm{M}$. Sabol, MD, PhD, St. Elizabeth Institute of Oncology, Heydukova 10 SK-812 50 Bratislava, Slovakia definition, as a POPF should be defined only clinically relevant condition. In the case of an increased amylase activity found in the fluid from an operatively placed drain without impact on the clinical outcome, no fistula should be reported. POPF ,grade A“by original definition from 2005 is nowadays no longer considered a true pancreatic fistula. It was replaced by the term "biochemical fistula” or „biochemical leak“ (BL) in literature. This BL has no impact on postoperative clinical course (3). On the contrary, a clinically significant POPF grade $\mathrm{B}$ and $\mathrm{C}$ requires an intensive management and surgical intervention, and percutaneous or endoscopic interventional drainage (ID) is often warranted.

In recent years, an interventional drainage (ID) has become the standard of care for symptomatic postoperative fluid collections. However it is not always evident whether POPF refers to grade B or C. Heidelberg group analysed the cohort of 2,955 patients, in which 403 patients developed POPF (13.6\%), $11 \%$ were grade A, $17 \%$ grade $\mathrm{B}$ and $72 \%$ grade $\mathrm{C}$. These patients underwent either ID or reoperation. There was a significant difference between these two groups concerning the hospital stay (33 days vs 47 days) and POPF-associated mortality ( $0 \%$ vs $37 \%, p<0.0001)$. According to their results, it was proposed to assign all the patients undergoing ID as POPF grade $\mathrm{B}$ and the patients requiring a reoperation should definitely remain within the category grade C (4). Similarly, in a situation when a grade B POPF leads to organ failure or to clinical instability or life-threatening haemorrhage requiring an ICU 
stay with an interventional endoscopic stenting or embolization fistula is considered as the grade $\mathrm{C}$. The largest global study of "grade C" POPFs following pancreaticoduodenectomy analysed 4.301 patients worldwide. In the American College of Surgeons National Surgical Quality Improvement Program (ACS-NSQIP), preoperative and intraoperative variables were compared between the grade C POPFs and non-grade C POPFs. Grade C POPFs developed in 79 patients (1.8\%) (5).

Concerning the fact, that POPF possesses a risk of mortality, many studies made effort to identify risk factors and early predictors for clinically relevant POPF (CR-POPF). Multicentre study carried out by the Japanese Society of Hepato-Biliary-Pancreatic Surgery evaluated the predictive factors associated with a clinically relevant pancreatic fistula grade B/C. Male gender, intraoperative bleeding $>1.000 \mathrm{ml}$, soft pancreas and drain amylase level on POD 1 of more than $4.000 \mathrm{IU} / \mathrm{L}$ were the significant predictive factors for CR-POPF. It seems very important to be able to predict whether the patient will develop CR-POPF in the early period after $\mathrm{PD}$, since these fistulas require changes in patient management and are associated with a higher mortality rate (6). Conversely, the authors of number of other studies reported that the amylase level in drainage fluid after PD had no clinical significance $(7,8)$. Interestingly, there was the pattern of pancreatic fistula defined by Pratt et al termed as a latent pancreatic fistula defined as initially lacking amylase-rich fluid, but ultimately becoming CR-POPF (9).

As determined by the number of multivariate analyses, the patients with CR-POPF were statistically more likely to have a small duct size, soft gland texture, pathology including ampullary, duodenal, cystic or islet cell neoplasms and an excessive intraoperative blood loss $(10,11)$. Grade $\mathrm{C}$ POPF, although infrequent and accounting for $15 \%$ of all POPF, represents a serious threat to the patient. Its overall incidence of $2.6 \%$ was reported in the patients undergoing Whipple procedures. Development of the grade $\mathrm{C}$ fistula may lead to death with a reported mortality rate up to $39 \%$ (12).

Moreover, the importance of CR-POPF lies in the fact that in addition to being a life-threatening complication, it prolongs hospitalization and increases hospital costs.

Most POPF can be managed non-operatively, but a significant number of grade $\mathrm{C}$ POPF do require a surgical intervention. The management of pancreatic fistula in the early period after PD is not sufficiently standardized. Surgical options include a peripancreatic drainage with a possible attempt of repair of the site of leakage, construction of a new pancreatic anastomosis, resection of the anastomosis with a remnant ligation, and a complete pancreatectomy (13).

\section{Comparison of stump closure techniques}

Generally, there are two widely used methods to accomplish pancreaticojejunostomy after pancreaticoduodenectomy: the invagination pancreaticojejunostomy or duct-to-mucosa pancreaticojejunostomy.

Several randomized controlled trials have been published in order to find out the best reconstruction method for PD. These high volume centres trials compared the invagination to duct-to-mucosa anastomosis or pancreatogastrostomy to pancreatojejunostomy (10-16). However, we have not obtained a clear consensus as to which reconstruction method is the best for pancreatic-enteric anastomosis. Reported data of POPF rates were 4-18\% in ductto-mucosa anastomosis, $3-15 \%$ in invagination, $8-25 \%$ in pancreatogastrostomy and $11-34 \%$ in pancreatojejunostomy $(14,15)$. The comparative studies of reliability of pancreaticojejunoanastomosis versus pancreaticogastroanastomosis found often discrepant results, but the recent systematic review and meta-analysis of randomized control trials showed no differences in the outcomes irrespective of the method of pancreatic anastomosis after PD (16). Discrepancies among the studies could result from many factors, as the type of pancreatic anastomosis or the use of pancreatic duct stent are currently not standardized across institutions. Also, an intraoperative drain placement varies according to institutions or consists at surgeon's discretion. Therefore, further investigations will be necessary to prospectively validate these predictive risk factors to confirm the possible relationship between these factors and the development of CR-POPF. The incidence of spontaneous resolution or response to medical treatment of various forms of pancreatic fistulas (high or low output fistula, pure or mixed fistula, end or side fistula) are different and often quite unpredictable. POPF commonly occurs following either PD or distal pancreatectomy (DP). The prevalence of POPF after a distal pancreatectomy in high-volume centres reaches approximately $30 \%$. The largest meta-analysis of all the major stump closure techniques during DP and resulting POPF rates suggests the superiority of stapler, combined stapler and suture closure, anastomosis of the pancreatic stump into the jejunum or stomach, and autologous patch application, when compared to a manual suture closure of the pancreatic stump. Furthermore, there seems to be no impact of laparoscopic versus open DP, spleen-preserving vs. spleen-resecting DP, and of sealants like TachoSil1, fibrin-like glues, or the stapler bio-reinforcement Seamguard1 on PF rates after DP (17). Zhang et al in the meta-analysis of 5.252 patients after DP identified the superiority of the combination of stapler and suture closure over suture closure alone. The use of stapler closure or anastomotic closure for the pancreatic remnant after a distal pancreatectomy significantly reduces POPF rates $(18,19)$. Neither stapler nor scalpel resection followed by hand-sewn closure of the pancreatic remnant during distal pancreatectomy showed any benefit compared to the other method in the terms of postoperative pancreatic fistula (20).

The reported incidence of POPF after a proximal pancreatoduodenectomy varies about 5-28 \% (21). Most of the large pancreatoduodenectomy series reported the rates of pancreatic fistula over $10 \%(22)$.

Proximal pancreaticoduodenectomy also incorporates the pancreatico-enteric anastomosis, which is associated with a lower average POPF rates (16\%) vs. approximately $31 \%$ for DP.

The risk factors related to POPF include: pancreatic texture, pancreatic pathology, high pancreatic juice output, pancreatic duct size and biochemical parameters. Numerous studies comparing various techniques of PJ failed to find a significant difference in POPF incidence among them. POPF can be often successfully 
treated by non-surgical means. Treatment of POPF is predominantly non-surgical. Endoscopic therapy and percutaneous treatments are important therapeutic modalities. Surgical intervention could be required in selected cases (23).

Generally accepted indications for pancreaticoduodenectomy have expanded to encompass a broad spectrum of periampullary tumours including both malignant and benign lesions, chronic pancreatitis and occasionally, trauma. During the last decade, although the perioperative mortality rate significantly decreased after pancreatoduodenectomy, the incidence of perioperative morbidity remains still high.

The results of the prospective randomized trial comparing pancreaticogastrostomy with pancreaticojejunostomy showed that the overall incidence of pancreatic fistula was $11.7 \%$, and the condition occurred with a similar frequency after pancreaticojejunostomy $(11.1 \%)$ and after pancreaticogastrostomy (12.3\%) (24). Numerous reports showed no clear evidence for or against one particular method of pancreaticoenteric anastomosis. The choice of pancreatic anastomotic method is often based on individual experience and adherence to basic principles such as: good exposure and visualization; fine, non-strangulating suture placement to produce a patent, watertight anastomosis and in the preservation of the blood supply $(25,26,27)$.

An undrained pancreatic fistula might be the most dangerous, especially, when recognized too late. Dutch Pancreatic Cancer Group performed a large study on the adherence to PRISMA guidelines evaluating the post-operative clinical, biochemical and radiologic variables for an early recognition of clinically relevant postoperative pancreatic fistula. In 37 studies comprising 8701 pancreatic resections (8603 pancreatoduodenectomies and 612 distal pancreatectomies), clinically relevant POPF occurred in 1532/8701 patients (18\%). The early prediction variables for early diagnosis of POPF included an elevated serum and drain amylase, non-serous drain efflux, positive drain culture, elevated temperature, elevated C-Reactive Protein and white blood cell count as well as peripancreatic collections on computed tomography (28).

Since the high rates of incidence of CR-POPF are reported in large centres, their prediction and early recognition is of paramount interest. Lower leukocytes and, particularly, lymphocytes can favour a suspicion of POPF after PD. An elevated CRP on the second postoperative day is considered an early biomarker of PF (29).

Some studies indicated that the incidence of POPF after DP is even higher than that of pancreaticoduodenectomy. In the recent Chinese single-centre retrospective analysis, the albumin difference level on postoperative day 1 was recognized as a new, accessible, objective predictor for POPF and might be helpful for the screening of high-risk patients. The rate of POPF was $15.64 \%$ $(33 / 211)$. This is the first study to identify albumin difference as an independent predictor of POPF after DP (30).

Most of the recent studies confirmed POPF independent risk factors such as: long operation time (more than $480 \mathrm{~min}$ ), intraoperative blood loss of more than $1000 \mathrm{ml}$, combined splenectomy, BMI $>25$, pancreatic thickness $(>15 \mathrm{~mm})(31)$.

Another retrospective study by Chang et al estimated POPF formation by measuring the thickness and sectional area of pan- creas with imaging technology taking into consideration an insufficient stapled closure during clamping (32).

On the other hand, the analysis of 157 patients after the distal pancreatectomy by Sivestri et al concluded that the pancreatic division level did not affect the rate of POPF (33).

Tissue texture of the pancreas and the diameter of the pancreatic duct considered as the risk factors for POPF after the proximal resection seemed not to be so apparent in the patients after the distal one. The abovementioned results can indicate that the mechanism of pancreatic fistula formation after these two resection procedures is different (34).

Lot of biomaterials are currently being used for closing the pancreatic stump like fibrin glue, synthetic gasket assisted reinforced staplers, mesh reinforcement of the staple line, polyglycolic acid (PGA) mesh, etc (35). However, RCT by Kondo et al failed to show any advantages of mesh reinforcement in terms of POPF over a stapled closure without mesh (36).

Although the effect of biological materials on the prevention of POPF remains still controversial, further research on improving the suture technology combined with new materials for the prevention of pancreatic fistula is still reasonable. A detailed instrumental/stapler management like a slow parenchymal flattening technique by Okano et al or prolonged peri-firing compression were described with the aim of avoidance of tearing of the pancreatic parenchyma during transection $(37,38,39)$.

An introduction of laparoscopic distal pancreatectomy revealed an issue of the stapler size selection, however Kim et al found that there is no suitable-sized cartridge for a pancreas thicker than $12 \mathrm{~mm}$ (40).

In the patients suitable for body/tail resection, the preoperative endoscopic pancreatic duct stenting was attempted assuming its protective impact on the reduction of POPF incidence (41).

In 2017 Heidelberg group completed the prospective clinical trial with an injection of a smooth muscle relaxant into the pancreatic duct before distal resection. The results were promising, authors concluded that this procedure could decrease the incidence of POPF after DP (42).

Once the POPF appears, the goal of treatment is a sufficient drainage. The resulting peritoneal fluid collection caused by pancreatic fistula could be usually effectively drained percutaneously under CT or ultrasound guidance (43).

With a sufficient drainage combined with an antibiotic therapy and nutritional support, most patients would recover uneventfully from POPF.

\section{Role of total pancreatectomy}

As it was mentioned, various techniques have been developed to deal with the pancreatic stump. Total pancreatectomy (TP) as one of them avoids pancreatointestinal anastomosis, it can also be considered an extension of oncological radicality in selected pancreatic cancer patients. The high local recurrence rates after Whipple resection and left-sided pancreatectomy could suggest that pancreatic cancer might develop multicentrically in the pancreatic gland, which makes TP an optimal choice. 
$541-546$

Widely accepted indications for TP include malignant pancreatic head tumours with the extension into the left part of the pancreas, locally advanced pancreatic head cancer, wide range changes in the pancreatic main duct, recurrent malignancy in the pancreatic remnant, rescue pancreatectomy with POPF in the emergency setting as salvage procedure to control sepsis, multifocal intraductal papillary mucinous neoplasm throughout the gland, multiple metastases of renal cell carcinoma or melanoma with no extra-pancreatic spread, multifocal neuroendocrine tumours including multiple endocrine neoplasia and hereditary pancreatic cancer. Also- a chronic pancreatitis in the setting of intractable pain (44).

International consensus guidelines for the management of intraductal papillary mucinous neoplasm (IPMN) recommend a resection for main duct disease with a clearance of all high-grade dysplasia (HGD). The association of the main duct IPMN with an extensive multifocal HGD represents a clear indication for TP (45). Besides ,standard“ total pancreatectomy, pylorus- and spleenpreserving total pancreatoduodenectomy (PpSpTPD) with a segmental resection of both splenic vessels has also been attempted in selected patients. We are the witnesses of expanding criteria for total pancreatectomy and it is probable that we will see more total pancreatectomies in future (46).

Pancreatectomy and autologous islet cell transplantation can be used as a salvage procedure in patients, where all other therapies failed. The results would be superior if performed earlier in the course of their disease. It is also a feasible management option for a complex pancreatic trauma in both adults and children (47)

\section{Conclusion}

There has been an update of The International Study Group of Pancreatic Fistula of definition and grading of POPF eleven years after its original definition. Treatment of POPF remains predominantly non-surgical. Endoscopic therapy and percutaneous radiologic interventions gained an important role among therapeutic modalities. However, in case of feared grade C POPF, reoperation is often necessary, almost in all affected patients being the only curative therapy and thus the treatment of choice (48).

A large number of different pancreatic anastomotic techniques have been applied by pancreatic surgeons globally, trying to minimalize the risk of POPF. The safest technique of the pancreatic stump closure remains an unsolved issue and an effective strategy for POPF prevention is still waiting for its unveiling. Currently used preventive measures directed to reduce POPF rate have been shown to be ineffective. An up-to-date meta-analyses of randomized controlled trials according to the newest 2016 ISGPS criteria also showed no significant difference in the rate of POPF in the PG group versus the duct-to-mucosa or telescope PJ group. Pancreaticogastrostomy is considered a safe alternative to pancreaticojejunostomy, especially in patients having very thin, non-dilated pancreatic duct and soft pancreas. According to the results of number of meta-analyses, PG and PJ seem to be comparable anastomotic techniques following $\mathrm{PD}$. The detailed analyses of various reconstructive techniques following pancreaticoduo- denectomy identified no difference between PJ and PG in the terms of risk of any surgical complication $(49,50,51)$.

Many experienced pancreatic surgeons in high-volume centres do consider a total pancreatectomy in selected cases with a high-risk pancreatic anastomosis and other contributing risk factors. In this respect, TP may serve as an alternative to a pancreatic anastomosis in an effort to eliminate sequels of possible POPF. This approach has been proposed recently, even in a conjunction to auto-islet transplantation (52).

A complete removal of the pancreas definitely eliminates the risk of POPF. However, resulting apancreatic state is associated with severe metabolic consequences. Total pancreatectomy is currently indicated in case of diffuse multicentric premalignant lesions, namely the "main-duct IPMNs" requiring a resection in every patient fit for surgery, when an entire duct is affected. Moreover, it can remove possible "skip lesions" of the main pancreatic duct that could be missed (53).

Another issue represents a salvage completion pancreatectomy for life-threatening POPF that also carries a high risk of postoperative morbidity and mortality.

Despite of well-established surgical procedures, new risk factors concerning a construction of pancreatic anastomosis have recently been emerged (54).

However, additional studies are needed to establish the most efficacious anastomotic technique after PD.

\section{References}

1. Bassi C, Dervenis C, Butturini G et al. Postoperative pancreatic fistula: an international study group (ISGPF) definition.Surgery $2005 ; 138: 8-13$.

2. Berger AC, Howard TJ, Kennedy EP et al. Does type of pancreaticojejunostomy after pancreaticoduodenectomy decrease rate of pancreatic fistula? A randomized, prospective, dual-institution trial. J Am Coll Surg 2009; 208: 738-747.

3. Bassi C, Marchegiani G, Dervenis C, Sarr M, Abu Hilal M, Adham M. The 2016 update of the International Study Group (ISGPS) definition and grading of postoperative pancreatic fistula: 11 Years After. Surgery 2017; 161 (3): 584-591.

4. Hackert T, Hinz U, Pausch T et al. Postoperative pancreatic fistula: we need to redefine grades B and C. Surgery 2016; 159: 872-877.

5. McMillan MT, Vollmer CM Jr, Asbun HJ et al. The characterization and prediction of ISGPF grade C fistulas following pancreatoduodenectomy. J Gastrointest Surg 2016; 20: 262-276.

6. Kawai M, Kondo S, Yamaue H, Wada K, Sano K, Motoi F et al. Predictive risk factors for clinically relevant pancreatic fistula analyzed in 1,239 patients with pancreaticoduodenectomy: multicenter data collection as a project study of pancreatic surgery by the Japanese Society of Hepato-Biliary-Pancreatic Surgery. J Hepato-biliary-pancreatic Sci 2011; 18: 601-608.

7. Reid-Lombardo KM, Farnell MB, Crippa S et al. Pancreatic anastomotic leakage after pancrea-ticoduodenectomy in 1,507 patients: a report from the PancreaticAnastomotic Leak Study Group. J Gastrointest Surg 2007; 11: 1451-1458

8. Shyr YM, Su CH, Wu CW, Lui WY. Does drainage fluid amylase reflect pancreatic leakage after pancreaticoduodenectomy? WorldJ Surg 2003; 27: 606-610. 
9. Pratt WB, Callery MP, Vollmer CM Jr. The latent presentation of pancreatic fistulas. Br J Surg 2009; 96: 641-649.

10. Pratt WB, Callery MP, Vollmer CM. Risk prediction for development of pancreatic fistula using the ISGPF classification scheme. World J Surg 2008; 32: 419-428.

11. Schmidt CM, Choi J, Powell ES et al. Pancreatic fistula following pancreaticoduodenectomy: clinical predictors and patient outcomes. HPB Surg 2009: 404-520.

12. Fuks D, Piessen G, Huet E et al. Life-threatening postoperative pancreatic fistula (grade C) after pancreaticoduodenectomy: incidence, prognosis, and risk factors. Am J Surg 2009; 197: 702-709.

13. Denbo JW, Orr WS, Zarzaur BL, Behrman SW. Towards defining grade $\mathrm{C}$ pancreatic fistula following pancreaticoduodenectomy: incidence, risk factors, management and outcome HPB 2012; 14: 589-593.

14. Bai X, Zhang Q, Gao $S$ et al. Duct-to-Mucosa vs Invagination for Pancreaticojejunostomy after Pancreaticoduodenectomy: A Prospective, Randomized Controlled Trial from a Single Surgeon. J Amer Coll Surge 2016; 222: 10-18.

15. Keck T, Wellner UF, Bahra M et al. Pancreatogastrostomy Versus Pancreatojejunostomy for Reconstruction After Pancreatoduodenectomy (RECOPANC, DRKS 00000767): Perioperative and Long-term Results of a Multicenter Randomized Controlled Trial. Ann Surg 2016V 263: 440-449.

16. Wente MN, Shrikhande SV, Müller MW, Diener MK, Seiler CM, Friess H, Büchler MW. Pancreaticojejunostomy versus pancreatico gastrostomy: systematic review and meta-analysis. Am J Surg 2007; 193 : 171-183.

17. Tieftrunk E, Demir IE, Schorn S, Sargut M, Scheufele F, Calavrezos L et al. Pancreatic stump closure techniques and pancreatic fistula formation after distal pancreatectomy: Meta-analysis and single-center experience. PLoS ONE2018; 13 (6): e0197553.

18. Diener MK, Knaebel HP, Witte ST et al. DISPACT trial: a randomized controlled trial to compare two different surgical techniques of DIStal PAnCreaTectomy - study rationale and design. Clin Trials 2008; 5 (5): 534-545.

19. Zhang H, Zhu F, Shen $M$ et al. Systematic review and meta-analysis comparing three techniques for pancreatic remnant closure following distal pancreatectomy. Br J Surg 2015; 102 (1): 4-15.

20. Probst P, Huttner FJ, Klaiber U et al. Stapler versus scalpel resection followed by hand-sewn closure of the pancreatic remnant for distal pancreatectomy. Cochrane Database Syst Rev 2015; 11: CD008688.

21. Alexakis N, Sutton R, Neoptolemos JP. Surgical treatment of pancreatic fistula. Dig Surg 2004; 21: 262-264.

22. Osada S, Imai H, Sasaki Y, Tanaka Y, Nonaka T, Yoshida K. Reconstruction Method After Pancreaticoduodenectomy. Idea to Prevent Serious Complications. JOP. J Pancreas (Online) 2012 Jan 10; 13 (1): 1-6.

23. Seetharam P, Gabriel Sunil Rodrigues. Postoperative Pancreatic Fistula: A Surgeon's Nightmare! An Insight with a Detailed Literature Review. JOP. J Pancreas (Online) 2015 Mar 20; 16 (2): 115-124.

24. Bassi C, Falconi M, Molinari E et al. Reconstruction by pancreaticojejunostomy versus pancreaticogastrostomy following pancreatectomy: results of a comparative study. Ann Surg 2005; 242: 767-773.

25. Lai EC, Lau SH, Lau WY. Measures to prevent pancreatic fistula after pancreaticoduodenectomy: a comprehensive review. Arch Surg 2009; 144: 1074-1080.
26. You D, Jung K, Lee H, Hero J, Choi S, Choi D. Comparison of different pancreatic anastomosis techniques using the definitions of the International Study Group of Pancreatic Surgery: a single surgeon's experience. Pancreas 2009; 38: 896-902.

27. Adams DB. The pancreatic anastomosis: the danger of a leak, which anastomotic technique is better? J Gastrointest Surg 2009; 13: 1182-1183.

28. Smits FJ, Molenaar IQ, Besselink MG et al. Dutch Pancreatic Cancer Group. Early recognition of clinically relevant postoperative pancreatic fistula: a systematic review. HPB (Oxford) 2020; 22 (1): 1-11. doi: 10.1016/j.hpb.2019.07.005.

29. Rodriguez-Lopez M, Tejero-Pintor FJ, Bailon-Cuadrado M et al. Impaired immune reaction and increased lactate and C-reactive protein for early prediction of severe morbidity and pancreatic fistula after pancreatoduodenectomy. Hepatobiliary Pancreat Dis Int 2020; 19 (1): 58-67. doi: 10.1016/j.hbpd.2019.05.003.

30. Jian Shen, Yushun Zhang, Jin Hu et al. Albumin difference as a new predictor of pancreatic fistula following distal pancreatectomy: a retrospective study of 211 consecutive patients. Langenbeck's Arch Surg (2020) 405: 55-62.

31. Li Jiang, Deng Ning, Xiaoping Che. Prevention and treatment of pancreatic fistula after pancreatic body and tail resection: current status and future directions. Front. Med 2019, https: //doi.org/10.1007/s11684-019-0727-3

32. Chang YR, Kang JS, Jang JY, Jung WH, Kang MJ, Lee KB, Kim SW. Prediction of pancreatic fistula after distal pancreatectomy based on cross-sectional images. World J Surg 2017; 41 (6): 1610-1617.

33. Silvestri M, Coignac A, Delicque J, Herrero A, Borie F, Guiu B, Fabre JM, Souche R. Level of pancreatic division and postoperative pancreatic fistula after distal pancreatectomy: a retrospective casecontrol study of 157 patients with non-pancreatic ductal adenocarcinoma lesions. Int J Surg 2019; 65: 128-133.

34. Martin AN, Narayanan S, Turrentine FE, Bauer TW, Adams RB, Zaydfudim VM. Pancreatic duct size and gland texture are associated with pancreatic fistula after pancreaticoduodenectomy but not after distal pancreatectomy. PLoS One 2018; 13 (9): e0203841.

35. Jang JY, Shin YC, Han Y, Park JS et al. Effect of polyglycolic acid mesh for prevention of pancreatic fistula following distal pancreatectomy: a randomized clinical trial. JAMA Surg 2017; 152 (2): 150-155.

36. Kondo N, Uemura K, Nakagawa $N$ et al. A multicenter, randomized, controlled trial comparing reinforced staplers with bare staplers during distal pancreatectomy (HiSCO07 Trial). Ann Surg Oncol 2019; 26 (5): 1519-1527.

37. Okano K, Kakinoki K, Yachida et al. A simple and safe pancreas transection using a stapling device for a distal pancreatectomy. J Hepatobiliary Pancreat Surg 2008; 15 (4): 353-358.

38. Nakamura M, Ueda J, Kohno H et al. Prolonged peri-firing compression with a linear stapler prevents pancreatic fistula in laparoscopic distal pancreatectomy. Surg Endosc 2011; 25 (3): 867-871.

39. Hirashita T, Ohta M, Yada K et al. Effect of pre-firing compression on the prevention of pancreatic fistula in distal pancreatectomy. Am J Surg 2018; 216 (3): 506-510.

40. Kim H, Jang JY, Son D et al. Optimal stapler cartridge selection according to the thickness of the pancreas in distal pancreatectomy. Medicine (Baltimore) 2016; 95 (35): e4441.

41. Hashimoto Y, Traverso LW. After distal pancreatectomy pancreatic leakage from the stump of the pancreas may be due to drain failure or pancreatic ductal back pressure. J Gastrointest Surg 2012; 16 (5): 993-1003. 
$541-546$

42. Hackert T, Klaiber U, Hinz $U$ et al. Sphincter of Oddi botulinum toxin injection to prevent pancreatic fistula after distal pancreatectomy. Surgery 2017; 161 (5): 1444-1450.

43. Dokmak S, Ftériche FS, Meniconi RL et al. A.Pancreatic fistula following laparoscopic distal pancreatectomy is probably unrelated to the stapler size but to the drainage modality and significantly reduced with a small suction drain. Langenbecks Arch Surg 2019; 404 (2): 203-212.

44. Andrén-SandbergA, Ansorge $\mathrm{Ch}$, Yadav TD. Are There Indications for Total Pancreatectomy in 2016 Dig Surg 2016; 33: 329-334.

45. Crosby J, Bellin MD, Radosevich DM et al. Gastrointestinal symptoms before and after total pancreatectomy with islet autotransplantation: the role of pancreatic enzyme dosing and adherence. Pancreas 2015; 44: $453-458$

46. Choi SH, Hwang HK, Kang CM, Yoon CI, Lee. WJ: Pylorus- and spleen-preserving total pancreatoduodenectomy with resection of both whole splenic vessels: feasibility and laparoscopic application to intraductal papillary mucin-producing tumors of the pancreas. Surg Endosc 2012; 26: 2072-2077.

47. Logue, JA, Scot III WE, Honkanen-Scott $M$ et al. Expanding the indications for pancreas resection and autologous islet cell transplantation (IAT) in both adults and children. HPB 2016; 18: e328-e329.

48. Luu AM, Krasemann L, Fahlbusch T, Belyaev O, Janot-Matuschek M, Uhl W, Braumann C. Facing the surgeon's nightmare: Incidence and Management of postoperative pancreatic fistulas grade $\mathrm{C}$ after pancreaticoduodenectomy based on the updated definition of the International Study Group (ISGPS). J Hepatobiliary Pancreat Sci. 2020 Jan 17.
49. Noor MA, Hanif F, Shakeel O, Bari H. Pancreaticogastrostomy: A Safe Option in Pancreaticoduodenectomy for Pancreatic Head and Periampullary Neoplasms. J Coll Physicians Surg Pak 2020; 30 (1): 51-56.

50. Yunxiao Lyu, Ting Li, BSc, Yunxiao Cheng, Bin Wang,Liang Chen, Sicong Zhao.Pancreaticojejunostomy Versus Pancreaticogastrostomy After Pancreaticoduodenectomy: An Up-to-date Meta-analysis of RCTs Applying the ISGPS (2016) Criteria. Surg Laparosc Endosc Percutan Tech 2018; 28: 139-146.

51. Coco D, Leanza S. Pancreaticojejunostomy vs Pancreaticogastrostomy Reconstruction Following Pancreaticoduodenectomy: Meta-Analysis of Various Techniques to Prevent Pancreatic Fistula after Duodeno-Pancreatectomy. Ann Digest Liver Dis 2018; 1 (1): 1005.

52. Balzano G, Maffi P, Nano R, Mercalli A, Melzi R, Aleotti F, Zerbi A, De Cobelli F, Gavazzi F, Magistretti P, Scavini M, Peccatori J, Secchi A, Ciceri F, Del Maschio A, Falconi M, Piemonti L. Autologous islet transplantation in patients requiring pancreatectomy: a broader spectrum of indications beyond chronic pancreatitis. Am J Transplant 2015; 16 : $1812-1826$

53. Del Chiaro M, Rangelova E, Segersvärd R, Arnelo U. Are there still indications for total pancreatectomy? Updates Surg 2016; 68 (3): 257-263.

54. Kim SG, Paik KY, Oh JS et al. The vulnerable point of modified blumgart pancreaticojejunostomy regarding pancreatic fistula learned from 50 consecutive pancreaticoduodenectomy. Ann Transl Med 2019; 7 (22): 630 .

Received February 3, 2020. Accepted March 16, 2020. 